\title{
Traceability in iron ore processing and transports
}

\author{
Bjarne Bergquist* \\ Quality Technology E Management, Luleå University of Technology, Luleå, Sweden
}

\section{A R T I C L E I N F O}

\section{Article history:}

Received 27 September 2011

Accepted 20 January 2012

Available online $\mathrm{xxxx}$

Keywords:

Modelling

Process control

Simulation

Mineral processing

\begin{abstract}
A B S T R A C T
The ability to track and trace products in a production process and in the transportation chain from supplier to customers is important for quality control and process improvements. However, good traceability is often difficult achieve for continuous process products as well as for batch produced products where batches intermix. In this paper, guidelines for improving traceability and setting up a traceability system in the iron ore production process are presented based on two case studies. One case is using process data in a simulation approach for a pellets plant and the other is using RFID methodology to trace pellets in the distribution chain. Results show that simulations can aid short-term traceability, whereas long term traceability is possible through marking pellets with RFID transponders, where the applications are suitable.
\end{abstract}

(c) 2012 Elsevier Ltd. All rights reserved.

\section{Traceability and quality}

It is a matter of survival to take actions to improve the offerings if the customers are not satisfied with the products they receive. To remain as a supplier, we may need to compensate our customers or even have to recall products at high costs. Thus, when customers have issues with the products, the producers quickly need to understand why the customers are unsatisfied and in what way their products are unsatisfactory to prevent larger damage. Failing equipment, human errors and variation of raw material properties often lead to product defects that may lead to harmful disturbances. Here, traceability concerns arises: if the traceability and data retrieval systems are good, it is possible to trace the path the product has taken through the production process or product delivery process to search for operational or material irregularities. Such irregularities could include defective raw material batches, different process settings, or unusual production events, for instance malfunctioning equipments such as mills, pumps or valves. Traceability systems coupled with prediction of when a product with analysed properties enters different sections could also aid process control when normal sampling is infrequent or laboratory analysis of samples is slow.

Creating traceability may be uncomplicated in processes where it is possible to mark individual products in early process steps, which for instance is the case for many types of parts production. Developments of the data storage and retrieval systems, barcode systems, or non-contact tagging systems such as radio frequency

* Address: Quality Technology, Department of Business Administration, Technology and Social Sciences, Luleå University of Technology, SE 97187 Luleå, Sweden. Tel.: +46920 492137.

E-mail address: bjarne@ltu.se identification (RFID), have made tracking and tracing systems available as off-the-shelf solutions for many types of production. The minerals processing industry experience larger difficulties when aiming to improve traceability.

This article describes the difficulties and possible solutions to these problems for obtaining good traceability for some continuous and semi-continuous production processes seen in minerals processing. The purpose is to give examples of how traceability can be improved in such process environments, and to outline steps that need to be taken to achieve better traceability. The paper starts by discussing traceability from a continuous process perspective, and then the paper focuses on two approaches for improving continuous process traceability, simulations based on flow type estimations and tracer experiments and an approach to use RFID.

\section{Continuous processes traceability}

Many products are not by themselves valuable enough to motivate the cost of an individually based marking system; other products cannot be marked in any physical way. With current technology, it is for instance not feasible to mark every little rock in a shipment of gravel or even the grains in a shipment of industrial diamonds or gold powder. Of course, liquids are the extreme examples. When products are in granular, liquid or slurry states, product mixing will occur when the product is handled, within the production process or when the product is transported. When the product uninterruptedly passes through different process steps, such as often is the case in the minerals processing industry, we thus face both the difficulty of having products continuously mixing and individual particles being too inexpensive to bear the cost of marking. Furthermore, the product may be subjected to 
aggressive environments such as high temperatures or pressures, and the product may change phases, such as from solids to liquids.

\section{Toward continuous process traceability}

Since we cannot recall and recollect the processing history of every particle or molecule in a continuous process, traceability becomes a statistical property rather than a deterministic. Customer complaints may, at best, be traced to an interval when it is likely that most of the problematic product passed different process sections. Traceability systems should thus have the possibility to follow or mimic how disturbances propagate through the product production and transportation chains. With this tracking ability, it is a matter of backward calculation to trace the origins of disturbances. It is also possible to calculate how to counteract disturbances and if such measures are necessary if the flow characteristics of the process and the correct countermeasures are known.

\section{Material flows in continuous production processes}

The flow characteristics of processes are important from traceability and prediction perspectives. Mixing will reduce the amplitude of product disturbances such as chemical variations, and mixing may be naturally occurring or an intended operation. On the other hand, such mixing will also prolong the time these disturbances are acting on the output. The disturbance propagation and control will be influenced if the products exhibit some degree of mixing at different product stages. The obtainable resolution of the traceability calculations is thus dependent on how detailed knowledge we have of the process flow, and of the process' product storage volumes.

The product flow through pipes or transports on conveyors only constitute a small part of the total time a product needs to travel through a chemical process, and flow measurement or estimations are usually sufficient for traceability purposes in such sections. However, chemical processes usually contain intermediate storages intended for mixing to achieve stable product properties over time. The processes may also include chemical reactors where the product needs to stay for the reaction to commence. If we are interested in when a given particle passes through a certain container or process, we need to know both the volumes of the containers and the current flow. The better our estimates of how media flow through the process section are, the better our estimates of time it remains in the section will be. Processes usually contain several sub-processes, storages or reaction containers. To calculate the residence time (the time an average particle spends within the system) of a process, we thus need to calculate the residence time of all sub-processes and containers. Naturally, large storage containers influences residence time more than process sections the particle passes quickly.

From a traceability outlook, we are interested in how disturbances entering a process section will propagate, and the mixing behaviour will influence the propagation. It may thus be insufficient merely to know the volume of the container and the average flow to and from it. The residence time is thus influenced by the design of storage or reaction containers, if containers have agitators to mix the volume, as well as by the properties of the flowing media. Process sections with the largest product volumes have also the largest effect on total process residence time.

\section{Modelling of flows for traceability}

Huda and Chung (2002) and Cox et al. (2006) suggest the use of simulation models to better understand the production process and to see the effect of different production scenarios in, for example, food manufacturing processes. Simulation models may also be used to achieve traceability in other continuous processes. However, simulations of continuous processes tend to focus on specific process sections and/or problems, see, for example, Finnie et al. (2005), and Bhaskar et al. (2007).

Often, coarse calculations of flow characteristics will suffice to reach a reasonable level of traceability. The simplest flow assumption is the plug flow model. Paper pulps consisting of networks of wood fibres exhibit a degree of shear strength which must be overcome before mixing occurs, and the same has been reported for flotation froth behaviour (Moys, 1978). Without agitators, such fluids tend to pass through containers or pipes without mixing. The stoves of blast furnaces are also designed to expose coke, limestone and ore charges to a gradual temperature increase and gas exposure before entering the hearth where it is smelted, and in many types of chemical reactors such as these, mixing is unwanted. Ideally, this flow system thus represents the other mixing extreme, the plug flow model, which implies that the matter entering a process section will remain unmixed through the section. Any disturbance entering a section exhibiting plug flow characteristics will be present to the same amplitude and duration in the exhaust, only delayed by the passage time. Hence, there is a fixed residence time for each infinitesimal volume of the flowing matter. Tubular reactors are examples where the plug flow approximation is often used. There are numerous examples of where plug flow assumptions are unsuitable. The perfectly mixed flow model may be a useful approximation of the material distribution within a container when liquids blend readily and process steps contain efficient agitators. The perfectly mixed flow suggests that the matter (fluid, powder etc.) entering a process section is instantly mixed with all matter residing in the section. Hence, in this type of flow, each infinitesimal volume of the section's content has the same properties (chemistry, temperature, microstructure, particle size etc) as any other volume of the container at any particular time. If the properties change, the change is equally fast in the whole volume.

If flows exhibit high degree of mixing in large containers, the amplitude of a disturbance will be greatly reduced. However, the disturbance will remain longer in the process which may be troublesome if the product is sensitive to that disturbance. A water plant taking fresh water from a large lake with a bacteria infection may for instance be worse off if the water of the lake is continuously mixed rather than demonstrating a plug flow between the fresh water inlet and exhaust.

All real flows will exhibit flow gradients, and the flowing material will therefore be mixed to some degree. The flows will thus in this sense be within the range between the plug flow and perfectly mixed flow approximation. Real flow systems also often include, for example, short-circuiting (bypassing) and stagnant regions (Fogler, 2005, p. 979). Slurries consisting of suspended solids in a liquid may segregate if the densities of the solid and liquid differ. The clay build-up resulting from particle sedimentation may quickly reduce the vessel volume (often called dead-zone) which will change the flow properties and reduce residence time of the flowing media.

In many minerals processes, other material than the product itself may constitute the bulk of the volume flow of processes. Slurries may contain less than $1 \%$ of the minerals at certain operations and gold concentrations in gold ores are often measured in $\mathrm{mg} / \mathrm{kg}$. Any modelling attempt needs to acknowledge the constituents of the modelled flows to predict the flow of the product.

Often, real-time process data are not available or cannot for other reasons be used for tracking and tracing products within processes. In these cases, process modelling may aid process control when products with known disturbance features are tracked through the processes, or for tracing products to where and when 
a process disturbance must have been initiated. The modelling step needs to be based on the prevailing conditions of the modelled processes such as, for instance, temperature, reaction speeds, flows, volumes, and concentrations. In simple cases, it may be possible to connect process events to product properties and create models from regular process data. All real systems have complicating factors, but sufficient precision for traceability purposes may, nonetheless, be reached through using ideal flow assumptions with addition of calibrating constants to account for short-circuiting or stagnant zones, for example. Off-line methods like time series analysis may be used in more difficult cases; see Vanhatalo et al. (2011).

The traceability accuracy of any modelling approach of a continuous process will depend on how accurately the model can predict the product flow. Finely meshed computational fluid dynamic models or discrete element methods for granular material have been used to simulate small process sections with continuous flows, see Bhaskar et al. (2007) and Finnie et al. (2005). Such models may be required to attain modelling results close to actual flow behaviour of, for instance, how chemical fluctuations propagate in the process. In practice, the complexities of these models often result in computational times and methodological difficulties that quickly become impractical. Reduced accuracy in favour of swiftness may prove adequate and the only viable solution. One approach using rougher estimates to create liquid food traceability using so called "virtual batch" calculations (see also below) for a dairy has been presented by Skoglund and Dejmek (2007).

\section{Tracers}

The flow of complex systems and sections are often possible to study experimentally by adding tracers to the input flow and monitoring the exhaust tracer concentration profile. We may for instance charge some grains of sand with radioactive elements so these grains can be detected through the process to generate data for estimation of the residence time distribution. This estimation may then be used as calibration of the process models, and to determine what type of flow that best characterises the system (plug the degree of mixing, how much of the product volume that is flowing or stagnant etc), see Fogler (2005). Observe, however, that the use of tracers relies on the assumption that the tracer particles behave the same way in the process as the product it is supposed to model. Tracers added to another component, such as adding water solvable tracers to slurries may not work to create ore traceability if the ore powder precipitates and exhibit grouts in stagnant regions.

\section{Batch traceability through RFID}

The RFID system consists of a transponder (the marker that carries information, typically a serial number), a reader and a system for data retrieval. The reader consists of an antenna and communication electronics, and the read information is then stored in a server, see Fig. 1.

RFID methodology has found its way into the minerals processing industry for many applications. Marking and tracking of equip-

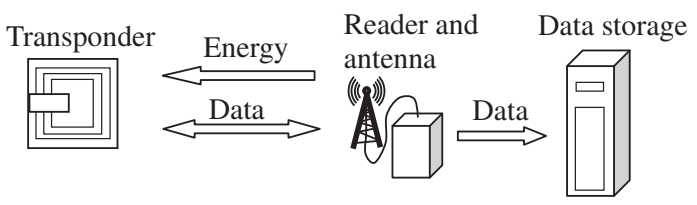

Fig. 1. RFID system. ment such as trucks are common applications, see e.g. (Gu et al., 2010).

RFID methodology has also been tested product traceability purposes, such as through tagging and tracking ore in real time. RFID transponders with special encapsulation have been inserted in drill holes before blasting, with only few transponders being lost even after crushing. The technology offers the mine possibility to customise production and crushing and concentrator operations to incoming ore grades (mineral/gangue ratio etc.). This ability to track ore grades is of particular importance if the ore may enter a plant from different mine fields (La Rosa et al., 2007).

RFID have been used for tracing granular material flows, where a fraction of iron ore pellets were marked with RFID transponders (Kvarnström and Vanhatalo, 2008).

\section{Traceability through creating virtual batches using RFID}

Batches are per definition not part of a continuous process, but it is sometimes convenient to divide the product flow into discrete objects such as batches. Such a virtual batch may be defined by the product passing a point in the process within an interval. These virtual batches may then be used to discuss, model or measure how products or disturbances propagate, even for complex flows.

An extension of the virtual batch concept is to add markers to the product at given intervals, and the RFID technique offers such possibilities (Kvarnström and Oghazi, 2008; Kvarnström and Vanhatalo, 2008).

Used as a traceability system, RFID transponders may mark the beginning and end of a virtual batch, and it is thereby possible to create traceability that does not solely have to rely on off-line calculations if the markers can be detected. Another effect of adding markers is that the spread and mixing behaviour exhibited between two points of the process can be estimated accurately. Knowledge of the mixing behaviour makes it possible to calculate dilution effects on disturbances.

The detectability of markers sets a limit to the type of products that are suitable. RFID transponders are manufactured as small as $50 \times 50 \mu \mathrm{m}$, so-called RFID dust, and transponders could thus be added to mineral powders, given that the transponders themselves are not a problem for the customers. However, since small transponders usually have lower read rates (Rao et al., 2005) and have shorter read ranges, small transponders may be hard to detect and the reader environment must be adapted accordingly.

\section{The case}

The main product of the Swedish mining company Luossavaara-Kiirunavaara $\mathrm{AB}$ (LKAB) is iron ore pellets (hereafter pellets). The company has two major production sites, Kiruna and Malmberget in the north of Sweden. The pellets production sites are located inland, and the product is transported first by train and then by boat from the harbours of Luleå and Narvik to customers mainly residing in Europe. The production and transportation processes contain continuous process steps as well as batch operations with differing batch sizes. The ore is excavated from deep underground pits, where every blast may be separated as a batch. The ore is then fed to a cobbing plant, where the ore is crushed, screened and, since the ore is ferromagnetic magnetite, the majority of the gangue minerals can be separated through dry magnetic separation. The cobbing plant works continuously, and the batchwise separation is thus lost in this step. The concentrator plant is also continuous and is located after the cobbing plant, where the ore is ground to a fine powder suitable for wet magnetic separation and for the next step of the continuous process, the pellets plant. The pellets plant includes steps to mix the ore powder with chemical additives before being rolled into spherical granules that are 
then sintered before shipment to customers. The finished pellet is an iron oxide ball of about $9-15 \mathrm{~mm}$ diameter containing more than $66 \mathrm{wt} \%$ iron. The process starting with the cobbing plant is continuous but with intermediate storages between plants. The distribution chain is both continuous and discontinuous, with conveyor systems, train transports, and boats constituting natural batches.

One point of departure of the paper is the transport of pellets between the pellets plant in Kiruna and the harbour of Narvik. The studied Kiruna-Narvik distribution chain includes three intermediate storage steps, a longer train transport and several shorter transports on conveyors between storages and the longer transports (Fig. 2). The production process and distribution chain together contain a mixture of continuous and batch flows, and can therefore be categorised as a semi-continuous process. The inflow to the buffer silos at the product plant is continuous, while the remaining flows are batch flows. The batch volumes of pellets going into or out of the process sections are determined by the buffer levels and arrival or departures of trains and boats. Traceability in the distribution process is further complicated by the design of some process steps, where the flow includes in the residence time of the granules.

The second point of departure is Malmberget where LKAB has two pellets plants. The plants are fed by two water/ore-powder slurries originating from different sources with differing iron ore properties (Fig. 3). The slurries are then mixed to ensure that both plants' pellets have matching properties. After the slurry mixing stage, the slurry is dewatered in the filter section, and the dewatered powder is then either temporarily stored or fed to a mixer where a binder is added. After the mixer, the powder flow is split into several streams and stored shortly before it is agglomerated and rolled into pellet granulates. The pellets are sieved after the rolling operation, and oversize pellets are crushed and returned to the mixing station, while undersize pellets are fed back to the rolling operation entry. The granulate streams from the different ball rolling machines are then merged and the granulated are sintered; the final treatment before distribution to the customer. The sintering furnaces also contain internal backflows, where $20 \%$ of the sintered pellets are fed back to the furnace entry to be used as a thermal protection for the equipment.

\section{Simulations to improve the ability to track and trace products}

An important quality characteristic of the pellet is stable chemical and physical properties. However, the company sometimes need to change the product properties for development purposes. The change of properties such as chemical composition in this process takes considerable time, and different products need to be kept separate due to quality concerns. Consequently, changes of product specifications interfere substantially with the regular production.

Moreover, the process lacks special arrangements for monitoring large shifts such as when product composition changes and it also lacks different storage facilities for different product types between the different process sections. The company has thus implemented a simulation model to control product changes and minimise the time when the process is between desired levels. The model is updated with current process data such as buffer levels, chemical analyses and product flows to calculate when the product enters different sections to aid plant control (Kvarnström and Bergquist, 2012). The model is developed in SIMULINK ${ }^{\mathrm{TM}}$ ver. 7.5 , which makes it possible to model the process in a graphical environment. The model is made up of process blocks representing the different process sections. The product flows between sections are represented by connection lines between the process blocks.

The model was initially based on blueprint volumes and coarse engineering estimates of the flow behaviours of the media in bins and storages throughout the plant, see Table 1 . The data in the table represent the equivalent plug flow residence times of the sections, i.e. the time it would take for the product to pass trough the section, given the section's volume and average flow. The engineering estimates of the flow mechanisms are also seen in Table 1.

However, initial analyses of the studied process showed that most residence time could be attributed to two storage types

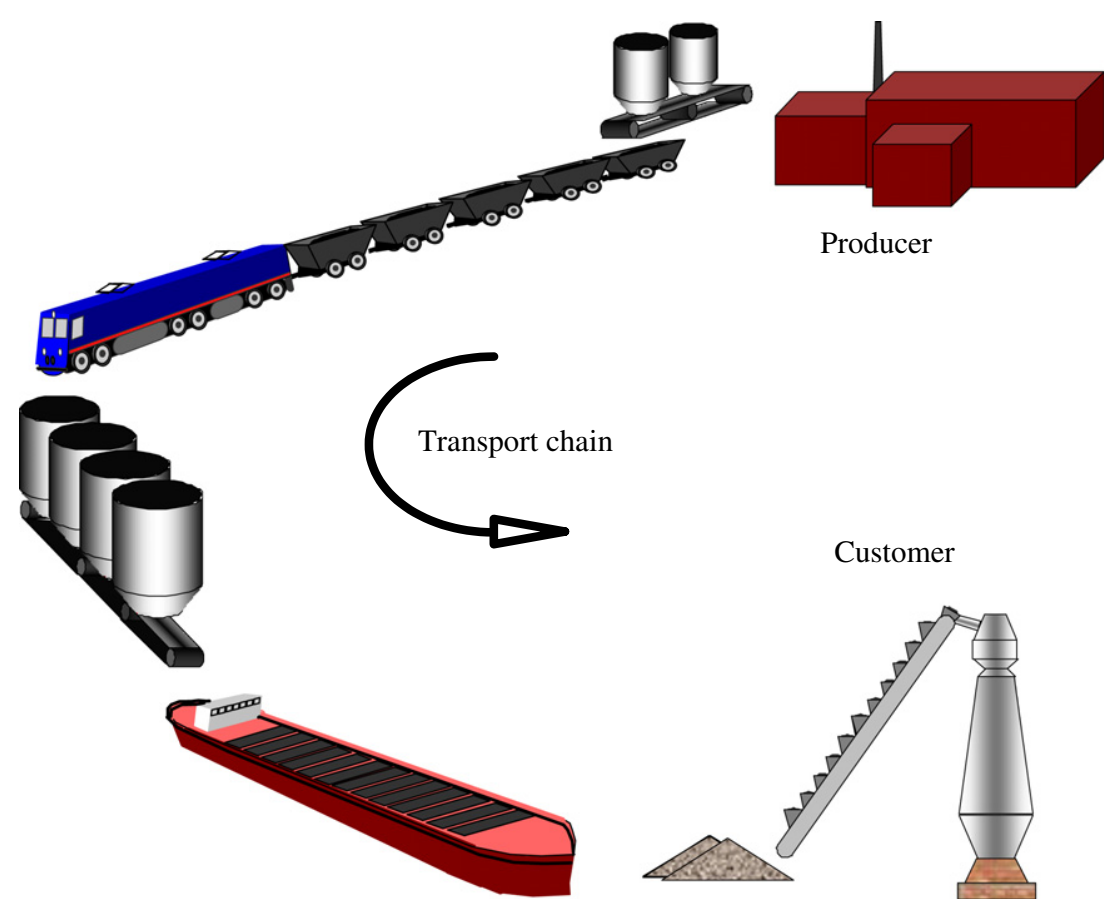

Fig. 2. The studied pellets distribution chain. 


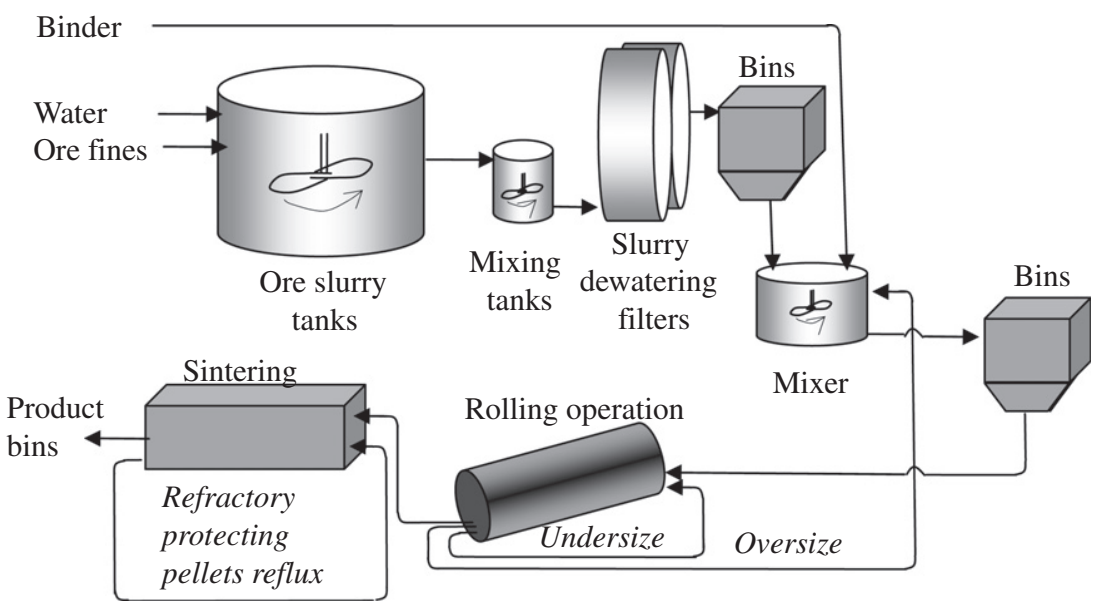

Fig. 3. Simplified flows and storages of one of the two pellets plants. There are also possibilities for cross-pumping between plants (not shown).

Table 1

Major process sections (Kvarnström and Bergquist, 2012).

\begin{tabular}{|c|c|c|}
\hline Process section & Equivalent plug flow residence time (\% of total time) & Estimated flow mechanism \\
\hline Slurry containers & 75 & Unknown \\
\hline Mixing containers & 0,2 & Perfectly mixed \\
\hline Filters & 0,5 & Perfectly mixed \\
\hline Ore powder storages & 7,4 & Unknown \\
\hline Mixers & 0,1 & Perfectly mixed \\
\hline Bins & 10 & Plug flow \\
\hline Balling granulators & 1,1 & Plug flow followed by perfectly mixed \\
\hline Sintering furnace & 5,4 & Plug flow with reflux \\
\hline
\end{tabular}

where flow properties were unknown, the slurry containers and the bins. By adding a tracer to the largest of these storages, the slurry containers, it was concluded that the flow could be approximated by an active volume with perfectly mixed flow, but also that the two containers had stagnant pockets of $25 \%$ and $39 \%$. These results were implemented in the simulation model. Two complementary models were designed; one with completely mixed flow in the bins and the other where bin flow was simulated using the other extreme, the completely mixed model. The simulation results showed that the models differed very little, and a bin tracer experiment was therefore not performed. The flow behaviours of the remaining containers were based on engineering estimates of flow behaviour (Kvarnström and Bergquist, 2012).

The final model can be used for offline prediction purposes, but can also be updated with current process data such as buffer levels, chemical analyses and product flows to calculate when the product enters different sections to aid plant control. If for instance an event at the concentrator or cobbing plants induces a $3 \mathrm{~h}$ disturbance, for instance that one of the magnetic separators for some reason starts to malfunction, such a disturbance can be modelled and it can be pinpointed when and for how long the disturbance will influence production downstream, and when to send the products back for rework. Fig. 4 shows an example where the effect of a disturbance of the ore chemistry of height 1 and duration of $3 \mathrm{~h}$ entering the slurry bins is studied. In Fig. 4, the extra amount of the unwanted chemical, e.g. gangue mineral, is studied in the pellets flow after one of the two sintering furnaces. The simulation is made assuming that all other conditions in the plant are kept constant.

\section{RFID marker tracers}

Simulation models strictly based on storage volumes and flows are not always possible means to create traceability, for instance

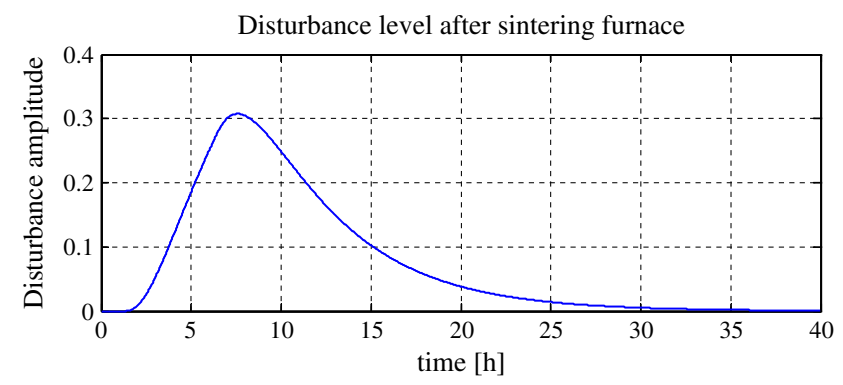

Fig. 4. Chemical disturbance level after one of the sintering furnaces, where 0 would represent an undisturbed process, and 1 would represent a fully disturbed process, i.e. that the chemical disturbance would be as severe as at the input to the slurry bins.

where storage volume measurements or estimations have large errors, or where product flows are not recorded. The distribution chain of iron ore pellets from the Kiruna production site to their customers around the world is an example of such a chain. Measurements of pellets volumes are often wrong and conveyors are started and stopped without coupling to a general control system. The suggested solution for this system was to add RFID transponders with properties resembling the pellets, either regularly or as an experiment as base for a statistical traceability model.

The distribution chain includes two longer transports by boat and train, three large intermediate storages, and many conveyor transports in-between. The process contains a mixture of continuous and batch flows, and was thus semi-continuous. At the plant, the inflow of pellets to the first silos is continuous, but after that, flows are batch-wise. However, these batch sizes vary depending on arrival or departures of trains and boats. Traceability in the distribution chain is further complicated by the design of some process steps, where the flow includes mixing. Process steps with 


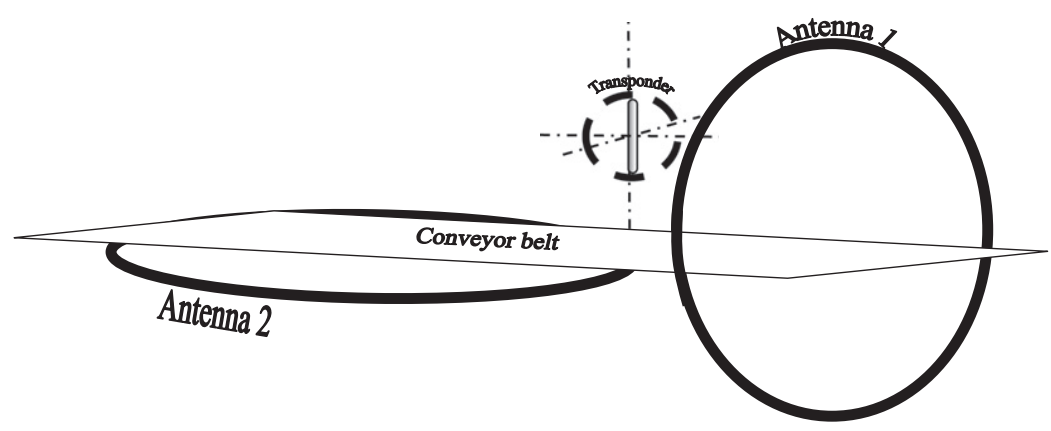

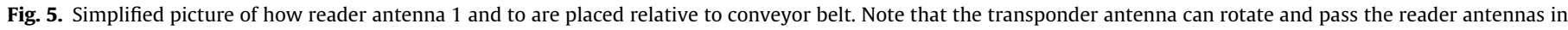
any direction.

intermittent flow induce residence time variation. The distribution chain was used to study if RFID markers could be used for creating virtual batches.

Important considerations have been transponder detectability, and two RFID readers were placed at conveyor belt positions after the product bin at the Kiruna plant and two readers were placed at the Narvik harbour. To use two readers at each location was motivated by increased detection probabilities. The antennas are direction sensitive, and since the orientation of the RFID-pellet antennas cannot be controlled in the product flow, orthogonally oriented reader antennas increases detection rates, Figs. 5 and 6.

Process residence times can be calculated with information from transponders of they have passed two known locations. These residence times can then be used for a simple statistical model of how the products propagate for offline use, simply by using the distribution of the residence times between two points. A more advanced solution would be to regularly drop RFID pellets into the product flow for continuous monitoring of the product. Such system would make it possible to track the product even when the process works irregularly, for example malfunctioning equipment such as train derailments or weather conditions cause delays and special product handling and storage.

Besides transponder detectability, another important consideration has been transponder encapsulation. To avoid risking transponders to segregate, and thus obtain other residence times than the products, the encapsulated transponders should have similar properties as the granular products they are to measure the residence time of. The sizes and densities, shapes and other properties of the granular media thus limit the RFID transponder types that are useful for the specific application. The pellets for this

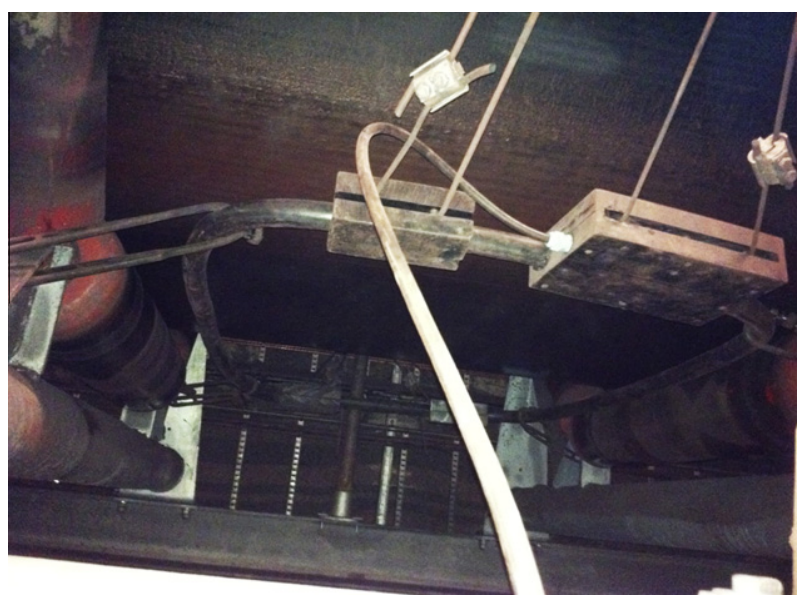

Fig. 6. Reader antenna located below conveyor belt. application are roughly spherical and have a diameter that range from 9 to $15 \mathrm{~mm}$. Initial tests showed that larger transponders than the pellets were much easier to detect than transponders that could fit within a casing the same size as pellets. Large efforts have thus been made to see if these larger transponders could be used without any segregation within the pellets flow. Both encapsulation geometry and density has been studied. In this experiment 86 large and 150 small RFID-pellets were inserted into the product stream in random order directly after the sintering furnace, to be detected by readers downstream. The larger number of small RFID-transponders was used to get enough readings also from the smaller types. The purpose of the experiment was to see if the RFID pellets containing larger transponders did have similar residence time as the smaller, and harder to detect types. The densities of the transponders was varied by adding led weights into the led-oxide composite encapsulation, see Fig. 7.

\section{Simulation results and discussion}

The first point of departure, the pellets production process, implemented a simulation model for their traceability needs. Using the simulation model, engineers could visualise the impacts of process disturbances. Since process settings continuously change during production, it is important that the simulation model does incorporate current flows and levels of silos, and an example of use of such model is seen in Fig. 8 (see also Kvarnström and Bergquist, 2012). The graph represents simulated and measured contents of a chemical additive that was changed during a field trial. The simulation results (solid line) are predicted product contents of the chemical after the pellets plant, and the dots are the obtained laboratory results. The simulation is based on initial settings in the factory, and continuously updated during the change of additive due to changed production schemes.

The method of using coarse estimations for flow behaviour, aided by small scale experiments and online measurements for calibration, has led to improved process predictions, and this should be true for many continuous processes, and not only those in the minerals processing industry. This kind of modelling may also be used to link existing process data from different locations and thereby create a process based data framework, see, for example, Skoglund and Dejmek (2007). The study also showed how process data coupled with simulation could increase the traceability of the product within a continuous process where normal laboratory analyses of samples are too slow for prediction purposes.

Mixing is one characteristic that differentiates continuous processes from discrete processes. Two effects of mixing were seen in the simulations: disturbances were smoothened and the impact of changes delayed. Mixing reduced the amplitudes of disturbances downstream, but the effect of the disturbance was also prolonged. In addition, the degree of mixing depends on the process and how 


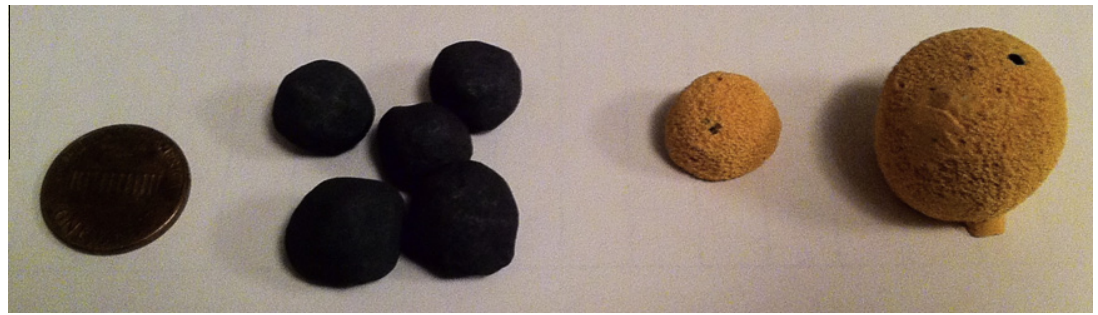

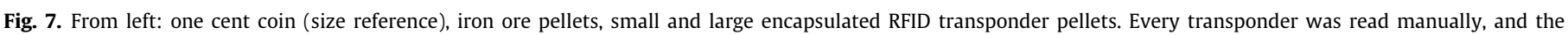
transponder number was noted before it was released into the product stream. All 236 pellets were inserted separately, but within 36 min during ongoing production.

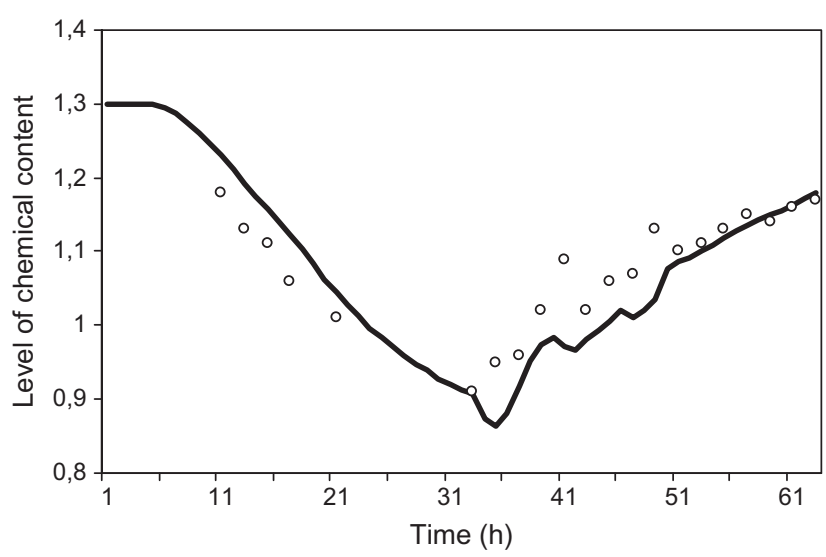

Fig. 8. Simulated chemical content after an intervention (solid line) and laboratory measurements (dots), based on results from (Kvarnström and Bergquist (2012) (published and modified with permission).

it is operated during the disturbance (volume of containers, speed of mixers are examples of process settings that will influence system response). The greatest tolerable duration and amplitude of a disturbance will vary depending on the sensitivity of downstream operations.

The control actions made in a process are usually based on various measurements. However, some properties may only be measured through laboratory analysis, and the laboratory analysis is usually too slow for most control purposes. We conclude that process simulations could be a decision support in such cases as the simulation can predict developing the property of interest.

Such as with any model, incorrect assumptions will affect the correctness in the output. For the studied process, the simulation model was based on existing process knowledge and calibrated using a small experiment, and the simulation results did, according to the engineers, correspond well with the measurements taken during the change. A simple model based on existing process knowledge may therefore be sufficient for continuous processes like the one studied here.

\section{RFID markers approach: results and discussion}

The first RFID pellets that were detected at the reader after the product bin storage appeared after $2 \mathrm{~h}$ and $50 \mathrm{~min}$. After an additional 26 min all but three RFID pellets that were to be detected had passed the reader. In total, 28 small RFID transponders were detected and 45 large transponders.

The original $36 \mathrm{~min}$ it took to insert all pellets into the product stream had thus shrunk to 26 min after the product bin, due to the larger capacity of the conveyor after the product bin storage. The correlation between when the pellets were inserted in the pellets flow and when they were detected was more or less nonexistent. Three additional transponders were again detected within $3 \mathrm{~min}$

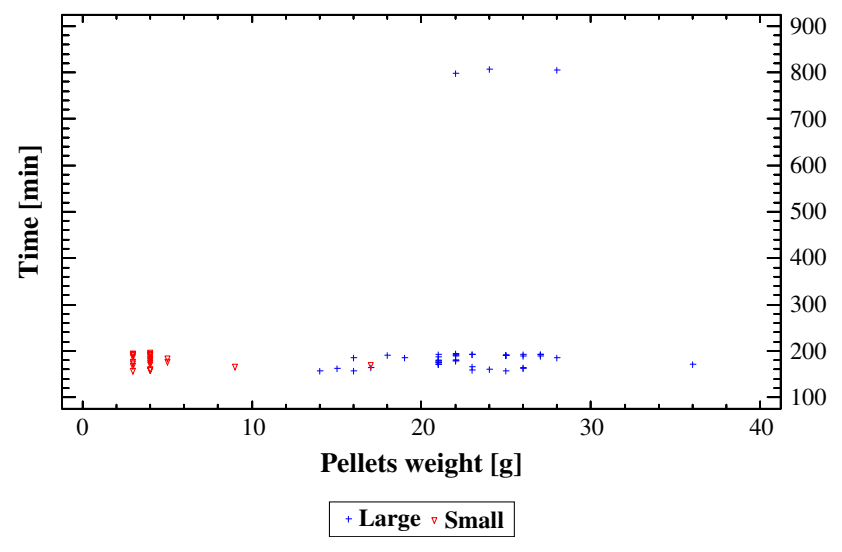

Fig. 9. RFID pellets' residence time. The resolution of the scale was $1 \mathrm{~g}$.

$11 \mathrm{~h}$ after the others, Fig. 9. Except for the three outliers, there was neither a correlation between pellets size and residence time, nor between pellets weight and residence time. There was not any correlation between when the pellets were dropped into the pellets flow and when they were detected, which would indicate that the pellets were mixed in the bins. The large deviation of the residence time of the outliers is likely due to stops off the conveyors.

Except for the three outliers, we did not find evidence that the larger transponders encapsulated in a casing with similar or higher density had different residence times, and thus segregated during these experiments. These larger transponders are therefore better suited for use as product batch delimiters since their read rate was significantly higher than that of the small transponders.

The other concern has been the equipment reliability. The environment where the readers have been placed, which is around pellets conveyors, is harsh. A common problem has been that conveyor belts have been interfering with antennas and other problems have been with difficulties to relay data from the readers. During this experiment, no data was obtained from the harbour readers, and the reason for this is not yet known.

\section{Conclusions, findings and future possibilities}

It is more difficult to create traceability in continuous processes, and traceability changes to a statistical property rather than a deterministic one. In this paper we have shown how tracers and modelling efforts may be used to improve the ability to track and trace products in continuous processes, which ultimately will improve quality control, product quality and reduce waste. For a general application, we suggest that many obstacles may be tackled beforehand by addressing the issues given below:

Ability to track products will aid process control. Often, minerals processing control will have to rely on measurements that are updated too seldom. Many properties are still measured through 
sampling and manual laboratory work. Laboratories are many times only open during regular working hours and new data may be available only once five times per week. Even if the laboratory personnel are working and laboratories are accessible, the laboratory sampling and measurement procedures may take much longer than what is required from a quality perspective. Products have already left the points where they could be accessed and traced when laboratory results are delivered to the process operators.

It is markedly easier to achieve good ability to track or trace products in industry branches where the producer does not need processing. The batch processing batches may be traceable through the process, if batches are kept separated through the process and transportation chains. When a batch sizes differ between storages or transports, or if continuous operations or operations where traceability is difficult to uphold such as in large warehouses or silos, the possibility for batch level traceability is lost. The process where the traceability system will be installed must thus be checked carefully so that critical events from a traceability perspective are identified. Since many minerals industries work with chemical reactions, processes are often harsh environments with respect to chemical aggressiveness of the product or of the processing environment, of temperatures and pressures. The most unforgiving environments, such as metal smelting operations may preclude other ways of improving traceability, than to make tracer experiments, for instance using radio active tracers (e.g. Towers et al. 1953), and to use the gathered information for computer modelling of the flow system within the process.

Some traceability systems are based on the inherent variation that the products exhibit, whereby a measurement of the profile of certain or several product properties may be used to determine the origin of the product. A famous example is traceability of nuclear materials using reference materials with known isotope contents (Donohue, 1998). A demand when using the property variation, or material signature, is of course that the properties vary enough to be detected, and that the detected variation can be linked to a known reference. Currently, only the most demanding applications can bear the costs measuring isotopes regularly on for instance raw material of a steel plant. Material signatures using particle texture analysis (Oghazi et al., 2009) have been tested for iron ore fines for traceability purposes, but many difficulties still need to be resolved.

\section{Acknowledgement}

We gratefully acknowledge the financial support from the Swedish mining company LKAB, Electrotech, VINNOVA (The Swedish Governmental Agency for Innovation Systems).

\section{References}

Bhaskar, K., Murthy, Y.R., Raju, M., Tiwari, S., Srivastava, J., Ramakrishnan, N., 2007. CFD simulation and experimental validation studies on hydrocyclone. Miner. Eng. 20 (1), 60-71.

Cox, R., Smith, J., Dimitratos, Y., 2006. Can simulation technology enable a paradigm shift in process control? Modelling for the rest of us. Comput. Chem. Eng. 30 (10-12), 1542-1552.

Donohue, D., 1998. Strengthening IAEA safeguards through environmental sampling and analysis. J. Alloy. Compd. 271-273 (12), 11-18.

Finnie, G., Kruyt, N., Ye, M., Zeilstra, C., Kuipers, J., 2005. Longitudinal and transverse mixing in rotary kilns: a discrete element method approach. Chem. Eng. Sci. 60 (15), 4083-4091.

Fogler, H., 2005. Elements of Chemical Reaction Engineering. Pearson Education, Upper Saddle River.

Gu, Q.-h., Lu, C.-w., Li, F.-b., Jing, S.-g., 2010. Mining production information management system in an open pit based on GIS/GPS/GPRS/RFID. J. Coal Sci. Eng. 16 (2), 076-181.

Huda, A.H., Chung, C.A., 2002. Simulation modelling and analysis issues for highspeed combined continuous and discrete food industry manufacturing processes. Comput. Ind. Eng. 43 (2), 473-483.

Kvarnström, B., Bergquist, B., 2012. Improving traceability in continuous processes using flow simulations. Prod. Plan. Control. 23 (5). doi:10.1080/09537287. 2011.561813.

Kvarnström, B., Oghazi, P., 2008. Methods for traceability in continuous processes: experience from an iron ore refinement process. Miner. Eng. 21 (10), 720-730.

Kvarnström, B., Vanhatalo, E., 2008. Using RFID to improve traceability in process industry - experiments in a distribution chain for iron ore pellets. J. Manuf. Technol. Manage. 21 (1), 139-154.

La Rosa, D., Valery, W., Wortley, M., Ozkocak, T., Pike, M., 2007. The use of radio frequency ID tags to track ore in mining operations. In: Magri, E.J. (Ed.), APCOM 2007, Department of Mining Engineering, University of Chile, Santiago, Chile.

Moys, M.H., 1978. A study of a plug-flow model for the flotation froth behaviour. Int. J. Miner. Process. 5 (1), 21-38.

Oghazi, P., Pålsson, B., Tano, K., 2009. Applying traceability to grinding circuits by using particle texture analysis (PTA). Miner. Eng. 22, 710-718.

Rao, K.V.S., Nikitin, P.V., Lam, S., 2005. Antenna design for UHF RFID tags: a review and a practical application. IEEE T. Antenn. Propag. 53 (12).

Skoglund, T., Dejmek, P., 2007. Fuzzy traceability: a process simulation derived extension of the traceability concept in continuous food processing. Food Bioprod. Process 85 (4), 354-359.

Towers, H., Paris, M., Chipman, J., 1953. Diffusion of calcium ion liquid slag. J. Met. 5 (11), 1455-1458.

Vanhatalo, E., Kvarnström, B., Bergquist, B., Vännman, K., 2011. A method to determine transition time for experiments in dynamic processes. Qual. Eng. 23 (1), 30-45. 